\title{
Journal of

\section{Biomimetic functionalization of porous substrates: towards model systems for cellular membranes}

\author{
Ingo Mey, ${ }^{* a}$ Claudia Steinem ${ }^{a}$ and Andreas Janshoff ${ }^{b}$ \\ Received 20th March 2012, Accepted 11th May 2012 \\ DOI: 10.1039/c2jm31737k
}

\begin{abstract}
In this review, membrane-functionalized pore arrays and pore-spanning membranes are revisited as versatile tools in biophysical research. Pore-spanning membranes can be generated on different pore arrays such as porous alumina and porous silicon substrates using appropriate surface functionalization strategies. Their mechanical properties can be readily determined by force indentation experiments revealing that the lateral tension of the membranes is strongly governed by the surface modification of the pore rims. Pore-spanning membranes separate two aqueous compartments, which makes them well-suited to study transport processes across these membranes and entrap molecules into the underlying atto- to pico-liter sized compartments. Besides pore-spanning membranes, membranes covering the entire porous area provide a large surface area for molecular recognition events making this system well-suited for biosensor applications and as an extraction unit for protein purification.
\end{abstract}

\section{Introduction}

Porous substrates are the basic materials for a relatively new class of biosensors and allow for the generation of artificial membrane model systems. ${ }^{1-14}$ Lazzara et al. ${ }^{14}$ have shown that porous alumina substrates, whose entire surface area is covered with a continuous lipid bilayer doped with a receptor lipid, are a versatile tool to study molecular recognition events between the receptor and a protein, which can be used in, e.g., protein purification. Membranes that suspend the pores of the porous material, the so-called pore-spanning membranes, provide a well-defined mimic of biological membranes, as they exhibit good long-term stability, while the separate two aqueous compartments. Based on pore-spanning membranes on highly ordered pore arrays, new biosensor applications have been developed. ${ }^{11,13}$ Mager and Melosh ${ }^{13}$ used pico- to attoliter-sized compartments sealed by pore-spanning lipid bilayers for the entrapment and triggered release of molecules. ${ }^{2,9}$ Like no other model membrane system, pore-spanning membranes reproduce properties of the whole cell cortex by providing a free-standing membrane, which is partially pinned to a regular mesh. ${ }^{7,8,15}$ As such, they allow us to study the properties of the cellular membrane such as transport and fusion processes. ${ }^{3,12,16,17}$ However, highly ordered pore arrays are not only used to attach artificial lipid bilayers and cellular membranes, ${ }^{6,18}$ but also to deposit graphene layers ${ }^{19,20}$ and polymer membranes ${ }^{10}$ to be able to elucidate their mechanical

${ }^{a}$ Georg-August University Göttingen, Institute of Organic and Biomolecular Chemistry, Tammannstr. 2, 37077 Göttingen, Germany. E-mail: imey@gwdg.de; Fax: +49 55139 14411; Tel: +49 551393095

${ }^{b}$ Georg-August University Göttingen, Institute of Physical Chemistry, Tammannstr. 6, 37077 Göttingen, Germany properties. The possibility to probe several hundred membranes at a time under the same conditions makes reproduction and statistical analysis of experimental data more reliable.

A great variety of materials are employed to fabricate regular arrays of open and closed pores with different pore sizes. Three of the most commonly used pore arrays are based on silicon oxide, silicon nitride, and aluminium oxide. ${ }^{1,21,22}$ Silicon substrates are generally utilized to produce pore arrays with pore diameters ranging from a few hundred nanometres up to several micrometres. Aluminium oxide can be optically transparent if nanopores ranging from 20 to $80 \mathrm{~nm}$ in diameter and interpore distances of roughly $100 \mathrm{~nm}$ are produced by anodic anodization. However, larger interpore distances and thus, pore diameters can also be formed by anodization of aluminium, so that the resulting aluminiumoxide substrates are optically non-transparent. ${ }^{21,22}$ Typical examples for pore arrays are given in Fig. 1A and B.

\section{Surface functionalization}

To generate pore-spanning membranes (Fig. 1D) or to set up biosensors based on molecular recognition, the surfaces of the porous substrates need to be appropriately functionalized (Fig. 1D). A number of strategies have been developed to selectively modify porous surfaces. Evaporation of metals like gold atop the porous substrate combined with gold-thiol chemistry is used to produce self-assembled monolayers (SAMs) ${ }^{1,2,7}$ on the pore rims, while silane chemistry can be applied on silicon and aluminium oxide surfaces. ${ }^{1,23-25}$ If only the upper surface of the porous substrate is covered with gold, the SAM functionalizes only the pore rims, while the pore interiors remain non-functionalized. In contrast, general silanization strategies 

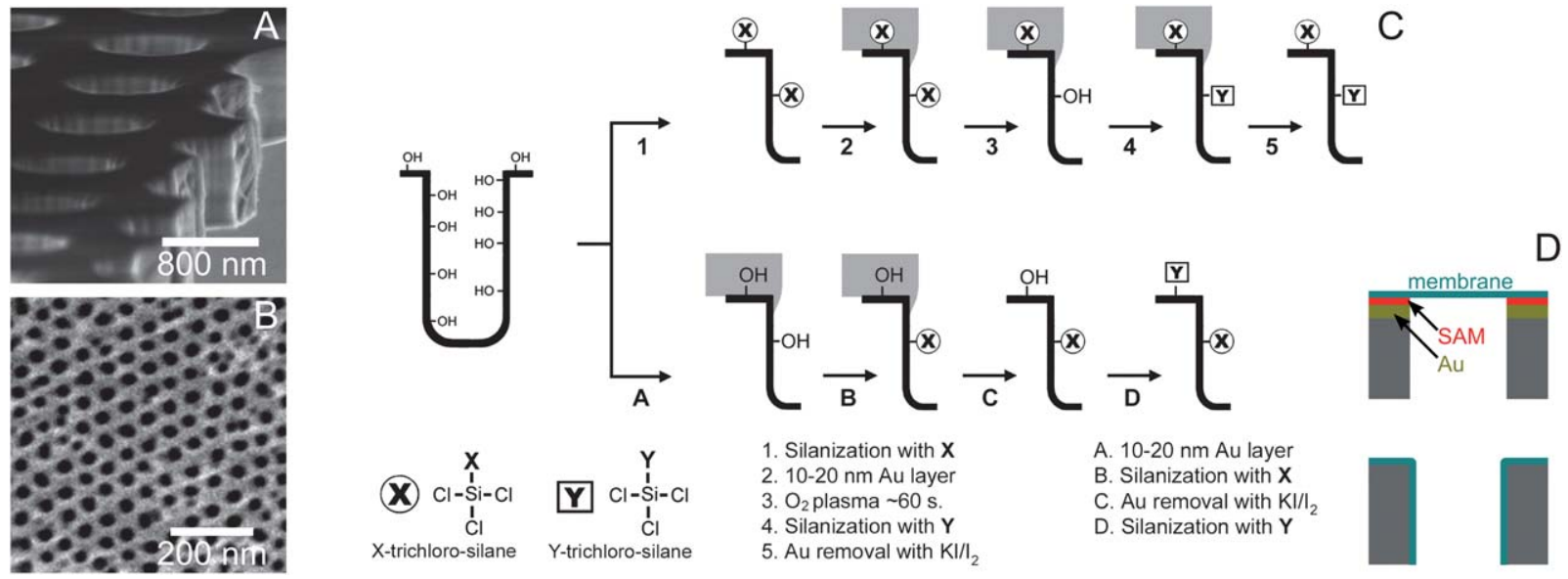

1. Silanization with $\mathbf{X}$

2. $10-20 \mathrm{~nm}$ Au layer

3. $\mathrm{O}_{2}$ plasma $\sim 60 \mathrm{~s}$.

4. Silanization with $Y$

5. Au removal with $\mathrm{KI} / \mathrm{I}_{2}$

A. $10-20 \mathrm{~nm}$ Au layer

B. Silanization with $\mathbf{X}$

C. Au removal with $\mathrm{KI} / \mathrm{I}_{2}$

D. Silanization with $Y$

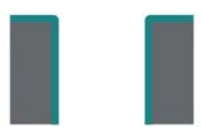

Fig. 1 Overview of different porous substrates and strategies for surface modification. (A) Micrometre sized pores in a $800 \mathrm{~nm}$ thick silicon nitride film with a pore diameter of $1.2 \mu \mathrm{m}$. (B) Regular pattern of pores in anodized aluminum oxide. ${ }^{23}$ (C) Two experimental protocols (1-5 and A-D) to prepare porous AAO substrates with dual functionalization using a thin Au layer as a protective mask (gray box). The final AAO substrates, after step 5 or step $\mathrm{D}$, have different pore-rim $v$ s. pore-interior functionalities. ${ }^{23}$ (D) The upper scheme visualizes a pore-spanning membrane while the lower one depicts a membrane covering the whole surface of a pore array.

functionalize the whole surface of the pore array. For the formation of pore-spanning membranes often a "rim-only" functionalization is advantageous, while modifying the entire surface results in membranes that cover the whole porous surface (Fig. 1D) ${ }^{23}$ Thus, strategies have been developed to orthogonally functionalize porous substrates by silane chemistry. ${ }^{26-29}$ However, most of the strategies have high failure rates or follow very complicated pathways. Lazzara et al. ${ }^{23}$ developed a new strategy to discriminate between the pore interior and the pore rims of pore arrays made of anodized aluminium oxide (AAO). To achieve the desired surface chemistries $(-\mathrm{X}$ and $-\mathrm{Y})$ a route of silanization, protection, removal and refunctionalization of the $\mathrm{Al}_{2} \mathrm{O}_{3}$ surface was developed as depicted in Fig. 1C. The basic idea is to use a thin gold layer as a protective layer for surface functionalization. While in step 1 the entire surface is modified by silanization $(-X)$, subsequently the pore rims are protected with a gold layer. By using $\mathrm{O}_{2}$ plasma in a third step the silanes on the inner pore walls are removed and the pore interior is prone to a second silanization step $(-Y)$. In a last step, the gold is removed to obtain an orthogonally functionalized surface. Another route starts by protecting the bare surface with gold and in the following steps, the remaining pore interior is functionalized $(-X)$, the gold removed and in a last step the pore rims are chemically modified $(-Y)$. This scheme is also applicable to silicon dioxide and silicon nitride surfaces. Such surface modification is the basis for porespanning membranes. ${ }^{25}$ The modification allows tuning of the lateral tension in the membrane, which might lead to an ideal mimicking of the mechanical properties of the plasma membrane. ${ }^{8}$ The functionalization strategies also pave the way for using porous substrates as selective and large surface areas. ${ }^{14}$

\section{Pore-spanning membranes}

A variety of materials like graphene sheets, ${ }^{19,20}$ cellular membrane fragments, ${ }^{6,18}$ protein membranes, ${ }^{9}$ lipid bilayers, ${ }^{1,2,7,8}$ and polymer membranes ${ }^{10}$ were prepared on pore arrays to investigate barrier functions comprising their electrical ${ }^{16,17}$ and mechanical properties ${ }^{2,7,30}$ as well as their possibilities to be used in sensor applications. ${ }^{11}$ In the next section, we will focus on pore-spanning membranes composed of well-defined lipids and lipid compositions. ${ }^{1-4,16,31}$

\section{Preparation of pore-spanning lipid membranes}

The various protocols for the preparation of pore-spanning lipid membranes lead to two different types of membranes, which we call hybrid pore-spanning membranes (Fig. 2A) and supported pore-spanning membranes (Fig. 2B). Hybrid pore-spanning membranes are based on hydrophobic self-assembled monolayers (SAMs) chemisorbed on gold-covered porous substrates. ${ }^{7,31}$ In these systems, a lipid bilayer is formed over the

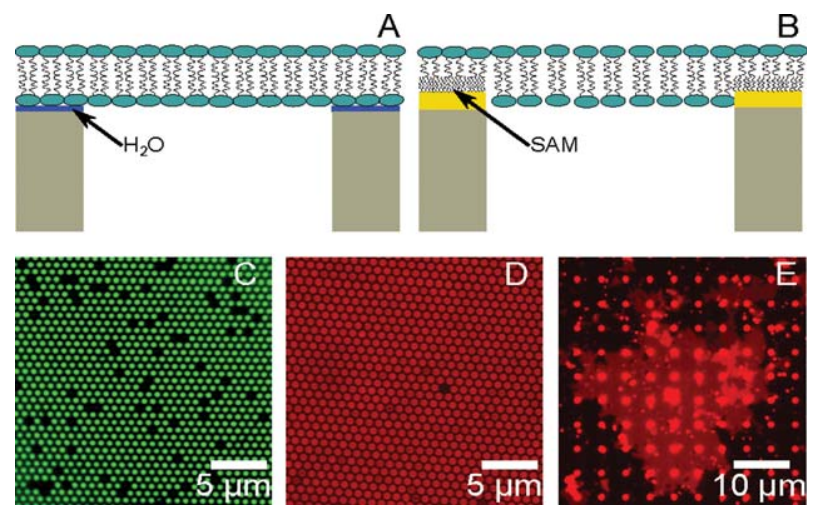

Fig. 2 Different systems of pore spanning lipid bilayers termed supported and hybrid pore-spanning membranes. (A) Illustration of supported porespanning bilayers prepared on hydrophilic porous substrates. The bilayer on the pore rims can slide reversibly into the pore upon indentation. (B) Illustration of a hybrid pore-spanning membrane. A gold layer on top of the substrate is equipped with a self-assembly layer prior to membrane deposition either via painting of lipids or spreading of giant liposomes. (C) Nano-BLMs composed of DPhPC/BodiPy-PC (99:1) on a CPEO3monolayer (pore radius: $400 \mathrm{~nm}$ ). (D) Nano-BLMs composed of DPPC/ TexasRed-DHPE (99: 1) on an OT-monolayer at room temperature (pore radius: $600 \mathrm{~nm}$ ). (E) Supported DPhPC/TexasRed-DHPE (99:1) bilayer on porous silicon with pore radii of $250 \mathrm{~nm}^{7}$ 
pores, while only a lipid monolayer is produced on the SAM. One preparation strategy is to spread phospholipids dissolved in $n$-decane on a hydrophobic SAM composed of, e.g., octanethiol (OT) or cholesterylpolyethyleneoxythiol CPEO3.7,16 Another approach results in solvent-free hybrid pore-spanning membranes by spreading of giant unilamellar vesicles (GUVs) on a hydrophobic monolayer composed of OT or CPEO3.,8,23 In contrast to the two previously described methods, supported pore-spanning membranes (Fig. 2A) are a continuous lipid bilayer. Here, hydrophilically functionalized porous substrates are used, on which GUVs are spread to form planar porespanning bilayers floating on a thin aqueous layer, which are solvent-free and comprise a symmetric lipid composition. ${ }^{\mathbf{1 , 2 , 8}}$

\section{Mechanical studies of pore-spanning lipid membranes}

The diversity of possible methods to study pore-spanning membranes ranges from optical methods like fluorescence microscopy ${ }^{13,15}$ to surface sensitive techniques like atomic force microscopy ${ }^{2,7}$ (AFM) and scanning ion conductance microscopy. ${ }^{32}$ These methods allow to analyse nearly all facets of membrane properties. To monitor the mechanical properties of membranes like bending modulus, tension and viscoelastic properties, pore-spanning membranes are localized by fluorescence and atomic force microscopy, and their mechanical properties are then assessed by force indentation experiments with high lateral resolution. The surface chemistry can be used to alter relevant properties of pore-spanning membranes such as the lateral tension as outlined below. ${ }^{8}$

Preparation of hybrid pore-spanning membranes yield longterm stable membranes and surface coverages of nearly $100 \%{ }^{13,16,31}$ as shown by fluorescence images (Fig. 2C and D), while for supported pore-spanning membranes patch formation is observed (Fig. 2E). The lateral diffusion of pore-spanning lipid bilayers can be analysed by fluorescence recovery after photobleaching (FRAP) experiments and fluorescence correlation spectroscopy. Supported and hybrid pore-spanning lipid membranes show a diffusion coefficient in the range of $10 \mu \mathrm{m}^{2} \mathrm{~s}^{-1}$ and low immobile fractions of $15 \%{ }^{8,24,31}$ These diffusion constants are roughly a factor of 4-10 times larger than diffusion constants found for solid supported membranes and match those found in giant unilamellar vesicles. The mechanical properties of such pore-spanning membranes have already been studied with great effort and success. In most cases, atomic force microscopy $(\mathrm{AFM})^{33}$ was utilized for imaging and indentation experiments. Fluorescence imaging allows the localization of a membrane patch and hence positioning of the AFM tip atop a membrane patch. To determine the mechanical properties, the membrane is centrally indented into the pore and the restoring force acting on the AFM tip is measured as a function of indentation depth (Fig. 3). ${ }^{2}$ In a physical model the membrane is described as a circular plate, indented in the center by a parabolic tip. Such a radial symmetric system can be reduced to the representation in Fig. 3.,34 Supported pore-spanning membranes give a soft response and can be indented several hundred nanometres into the pore, while hybrid pore-spanning membranes show a stiff response and a clear rupture event after several ten nanometres of indentation (Fig. 4A). Lysis of hybrid pore-spanning membranes is conceivable as the bottom layer of the suspended

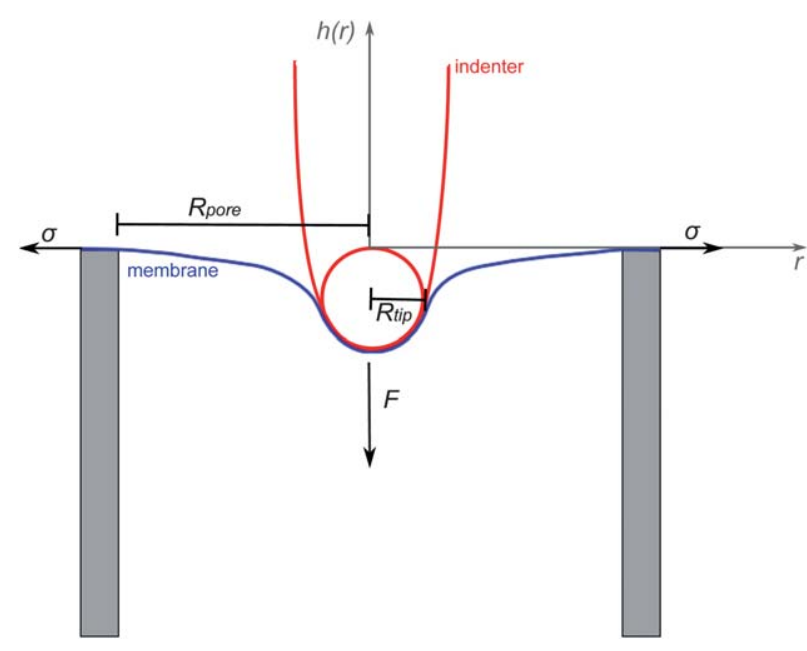

Fig. 3 Model for the description of a parabolic tip poking into a circular membrane with radius $R_{\text {pore}}, \sigma$ the lateral tension, $R_{\text {tip }}$ the radius of curvature of the tip, $F$ the normal force exerted by the tip, and $h(r)$ the shape of the membrane as a function of displacement from the center of the pore. ${ }^{2}$

bilayer has no reservoir of lipid material to access upon indentation and lipid bilayers can only resist an area increase of approximately $5 \% .^{7,32}$ Neither for supported pore-spanning lipid membranes nor for hybrid systems was a hysteresis between indentation and retraction found, indicating a purely elastic response upon indentation. This is further confirmed as the response of the lipid bilayers subjected to indentation exhibits no dependency on the indentation velocity ruling out viscoelastic responses due to the low surface viscosity of the lipid bilayers. ${ }^{2,7,8}$ Indentation of pore-spanning lipid membranes shows a predominantly linear response, which is mainly attributed to pre-stress. This is supported by the observation that indentation of hybrid pore-spanning membranes leads to lysis well below $5 \%$ area dilation..$^{7,35}$ Molecular dynamics simulations of pore-spanning membranes also showed pre-stress and early lysis upon indentation. ${ }^{36}$ The slope of the elastic response is determined by linear regression as a first measure for membrane properties. It is interpreted as an apparent spring constant $k_{\text {app }}$ for the membrane, but besides contributions from membrane properties it also depends on the substrate geometry. ${ }^{2,7}$ Force indentation curves on DPhPC lipid membranes spanned over pores with different radii plotted in Fig. 4B show a decrease of $k_{\text {app }}$ with increasing pore radius. ${ }^{7}$

To elucidate the mechanical properties of pore-spanning membranes three major contributions have to be considered: lateral tension, bending, and stretching. ${ }^{18,30,34}$ Stretching of the membrane occurs because during indentation the membrane area increases ${ }^{9}$ and bending energy has to be spent upon curving of the membrane. Lateral in-plane tension arises since the membrane adheres to the non-porous part of the silicon substrate thus generating excess of free-energy. ${ }^{7}$ During indentation the membrane essentially flows into the pore and resistance to the accompanying area increase is due to the associated tension. Prestress and stretching can be cast into a lateral tension $T$ :

$$
T=\sigma+K_{\mathrm{A}} \frac{\Delta A}{A_{0}} .
$$



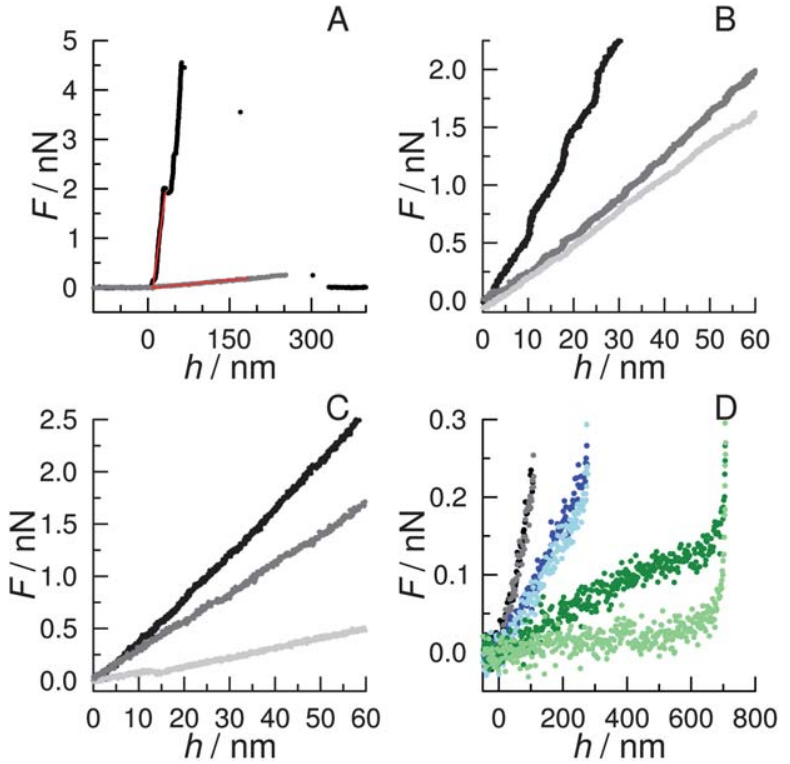

Fig. 4 Force-indentation curves at the beginning on pore spanning lipid membranes showing influences of the substrate and lipid composition. (A) Force indentation curves obtained from a DPhPC-tetradecanethiol hybrid membrane (black) and on a supported pore-spanning POPC membrane (gray) together with the corresponding linear regression to determine the slope $k_{\text {app: }}$ (black) $20.4 \pm 0.2 \mathrm{mN} \mathrm{m}^{-1}$ and (gray) $1.0 \pm 0.4$ $\mathrm{mN} \mathrm{m}^{-1}{ }^{8}$ (B) Dependence of the apparent spring constant on the pore radius, black: $R_{\text {pore }}=225 \mathrm{~nm}$, gray: $R_{\text {pore }}=400 \mathrm{~nm}$, light gray: $R_{\text {pore }}=600$ $\mathrm{nm}$. All force indentation curves were recorded on DPhPC hybrid porespanning membranes on an octanethiol SAM. ${ }^{7}$ (C) The elastic response of a DPPC membrane in the fluid (light gray) and gel phase (black) accompanied by a mixture DPPC-cholesterol (7:3) (gray) showing differences in response as a function of the physical state and composition of the membrane. (D) Typical force indentation curves obtained on (gray and black) a pure POPC bilayer and on the same POPC bilayer in the presence of 2-propanol (blue, light blue: $0.67 \mathrm{M}$ and green, light green: $1.28 \mathrm{M}$ ). Hysteresis between the indentation (dark color) and relaxation (light color) curves becomes more apparent in presence of 2-propanol. ${ }^{8}$

Where $K_{\mathrm{A}}$ is the area compression modulus and $\Delta A / A_{0}$ is the relative area increase upon indentation. By assuming a homogeneous lateral tension an analytical expression between indentation depth $h$ and external force $F$ is found: ${ }^{34,37}$

$$
h=\frac{F}{4 \pi \sigma}\left[1-\ln \left(\frac{F R_{\mathrm{tip}}}{2 \pi \sigma R_{\mathrm{pore}}^{2}}\right)\right] .
$$

Stretching of the membrane is resembled by a non-linear spring which depends in a cubic manner on the indentation depth, pore radius $R_{\text {pore }}$, tip radius of the indenter $R_{\text {tip }}$, thickness of the membrane $d$, and the elastic modulus $E^{38}$

$$
F(h)=\frac{9 \pi E d}{16 R_{\text {tip }}^{2}\left(\frac{R_{\text {pore }}}{R_{\text {tip }}}\right)^{9 / 4}} h^{3}
$$

Considering the linear and cubic dependencies in eqn (2) and (3) the shape of force indentation curves obtained on lipid membranes suspended over large apertures demonstrates that lateral tension is the governing process. In Table 1 the lateral tension for pore-spanning membranes composed of different lipid compositions and prepared on different surfaces with different pore geometries are summarized. Hybrid pore-spanning membranes display high tensions around $10 \mathrm{mN} \mathrm{m}^{-1}$ while supported pore-spanning membranes exhibit tensions below 1 $\mathrm{mN} \mathrm{m}{ }^{-1}$. Notably, the residual solvent leads to a decrease in membrane tension as shown by different preparations for hybrid pore-spanning bilayers made from DPhPC. Tension in porespanning membranes depends on surface modification but subtle differences can also be measured for different membrane compositions and the physical state of the membrane.

Small volume fractions of alcohols like 2-propanol are known to modulate the tension of lipid bilayers. ${ }^{8,39}$ Force indentation curves of pure POPC supported pore-spanning membranes and POPC membranes treated with different concentrations of 2propanol are shown in Fig. 4D. With increasing concentration of 2-propanol the apparent spring constant decreases, which indicates the decreasing tension of the membrane. Also a hysteresis between indentation and relaxation becomes visible with an increasing concentration of 2-propanol. This energy dissipation can be attributed to increased interbilayer friction as intercalation of the two leaflets takes part and 2-propanol is inserted into the bilayer upon indentation. In summary, geometry, surface chemistry, and especially interaction of membrane and substrate determine the membrane's response upon indentation. Since the mechanical response of supported pore-spanning lipid membranes on large pores are governed by pre-tension as also found in suspended graphene layers, ${ }^{7,20}$ the exact determination of membrane tension is readily accessible. Gonçalves et al. prepared pore-spanning S-layer membranes from Corynebacterium glutamicum and found that protein membranes are mainly

Table 1 Summary of results obtained for a variety of pore-spanning membranes $^{h}$

\begin{tabular}{lllcl}
\hline Lipid & $R_{\text {pore }} / \mathrm{nm}$ & Surface & $k_{\text {app }} / \mathrm{mN} \mathrm{m}^{-1}$ & $\sigma / \mathrm{mN} \mathrm{m}^{-1}$ \\
\hline DPhPC $^{a}$ & 225 & OT & $48 \pm 15$ & $22 \pm 6$ \\
$\mathrm{DPhPC}^{a}$ & 400 & OT & $36 \pm 8$ & $21 \pm 4$ \\
DPhPC $^{a}$ & 400 & CPEO3 & $26 \pm 5$ & $15 \pm 3$ \\
DPhPC $^{a}$ & 600 & OT & $25 \pm 6$ & $18 \pm 3$ \\
DPPC $^{a}$ & 600 & OT & $40 \pm 4$ & $31 \pm 3$ \\
DPPC/Chol $^{a}$ & 600 & OT & $31 \pm 4$ & $23 \pm 3$ \\
DPPC $^{a, b}$ & 600 & OT & $9 \pm 2$ & $7 \pm 1$ \\
DPPC/Chol $^{b}$ & 600 & OT & $14 \pm 3$ & $11 \pm 3$ \\
DPPC $^{b}$, & 600 & OT & $21 \pm 3$ & $16 \pm 2$ \\
DPhPC $^{c}$ & 600 & OT & $34 \pm 5$ & $26 \pm 4$ \\
DPhPC $^{d}$ & 250 & SiO & $0.21 \pm 0.05$ & $0.15 \pm 0.05$ \\
POPC $^{f}$ & 600 & ME & $0.9 \pm 0.3$ & $0.6 \pm 0.2$ \\
POPC $^{g}$ & 600 & ME & $0.3 \pm 0.1$ & $0.19 \pm 0.07$ \\
POPC $^{c}$ & 600 & ME & $2.6 \pm 0.12$ & $2 \pm 0.09$ \\
DOPC $^{c}$ & 600 & ME & $1.42 \pm 0.02$ & $1.02 \pm 0.01$ \\
DOPC/Chol $^{c}$ & 600 & ME & $3.82 \pm 0.16$ & $3.5 \pm 0.15$ \\
DPPC & 33 & ME & $370 \pm 100$ & - \\
DPPC $^{b, e}$ & 33 & ME & $48 \pm 15$ & - \\
DPhPC $^{e}$ & 33 & ME & $81 \pm 17$ & -
\end{tabular}

${ }^{a}$ Pore-spanning membrane containing solvent. ${ }^{b}$ At $50{ }^{\circ} \mathrm{C} .{ }^{c}$ Spreading of GUVs on chemisorbed thiols on gold-covered porous substrates. ${ }^{d}$ Spreading of GUVs on clean porous silicon dioxide samples. ${ }^{e}$ Spreading of LUVs on chemisorbed thiols on gold-covered porous substrates. ${ }^{f}$ In presence of $0.67 \mathrm{M}$ 2-propanol. ${ }^{g}$ In presence of $1.28 \mathrm{M}$ 2-propanol. ${ }^{h}$ Abbreviations used for the lipids: 1,2-diphytanoyl-snglycero-3-phosphocholine (DPhPC), 1,2-dipalmitoyl-sn-glycero-3phosphocholine (DPPC), cholesterol (Chol), 1-palmitoyl-2-oleoyl-snglycero-3-phosphocholine (POPC) and 1,2-dioleoyl-sn-glycero-3phosphocholine (DOPC). 
governed by stretching. ${ }^{9}$ They were able to determine an elastic modulus and could calculate the bending rigidity to be $90 k_{\mathrm{b}} T$ for the S-layer membrane. For pore-spanning lipid membranes, bending becomes relevant only if the pore size is small, but small pores are not ideally suited to determine the intrinsic bending modules of lipid bilayers, since the response of fluid bilayers to indentation compiles the various contributions from lateral tension, stretching, and bending. In general, this would not pose a principal problem if the indentation depth would not be limited geometrically to only a few tens of nanometres. The limited indentation depth generates force indentation curves that are just linear, which does not allow extraction of more than one independent parameter.

A solution to this inherent problem is provided by employing a different strategy to impose a curvature based free energy penalty on the system during indentation. By recording a force cycle of indentation and retraction as illustrated in Fig. 5A and B, the bending modulus and lateral tension of a membrane can be determined. The method relies on a combination of bilayer indentation upon approach of the cantilever, providing the lateral tension $\sigma$ and subsequent tether formation upon retraction, from which the bending modulus $\kappa$ can be inferred if $\sigma$ is known. ${ }^{6} \mathrm{~A}$ typical force cycle obtained from indenting a supported porespanning bilayer followed by pulling a membrane nanotube (tether) out of the membrane is shown in Fig. 5C. The jump to the baseline corresponds to a stochastic rupture of the tether. The force to form such a membrane nanotube $F_{\text {tether }}$ is related to the product of lateral tension $\sigma$ and bending modulus $\kappa$ according to eqn (4), neglecting viscoelastic contributions:

$$
F_{\text {tether }} \approx 2 \pi \sqrt{2 \sigma \kappa} \text {. }
$$

Fig. 5D shows two normalized histograms of the lateral tension and the tether force obtained from $n>50$ force cycles on pure pore-spanning POPC bilayers.

The feasibility of the approach to locally determine the bending modulus of free-standing membranes was demonstrated by using two different lipid compositions that exhibit different bending moduli without entering the gel phase. Pore-spanning membranes composed of POPC/cholesterol $(3: 1)$ exhibit both, a larger lateral tension and a larger tether force compared to pure POPC bilayers. From $\sigma$ and $F_{\text {tether }}$ the bending moduli were computed according to eqn (4). For a pure POPC bilayer we determined an average bending modulus of $(6.7 \pm 2.3) \times 10^{-20} \mathrm{~J}$. This value is in good agreement with the literature values ranging from 0.77 to $1.6 \times$ $10^{-19} \mathrm{~J}$ while for the POPC-cholesterol mixture an increased bending modulus of $(8.7 \pm 3) \times 10^{-20} \mathrm{~J}$ was found. ${ }^{40,41}$

In nature, membrane properties are tuned by lipid composition and connection between the membrane and cytoskeleton employing several hundred different proteins and lipids. This makes a direct copy of natural membranes rather complicated. Polymeric membranes are therefore in discussion as a substitute for lipid membranes e.g. in drug delivery applications. ${ }^{42}$

\section{Pore-spanning polymer membranes}

Synthetic amphiphiles like diblock-copolymers are commonly used as model systems for biological membranes. ${ }^{42,43}$ The lipidlike hydrophilic/hydrophobic proportions are able to self-
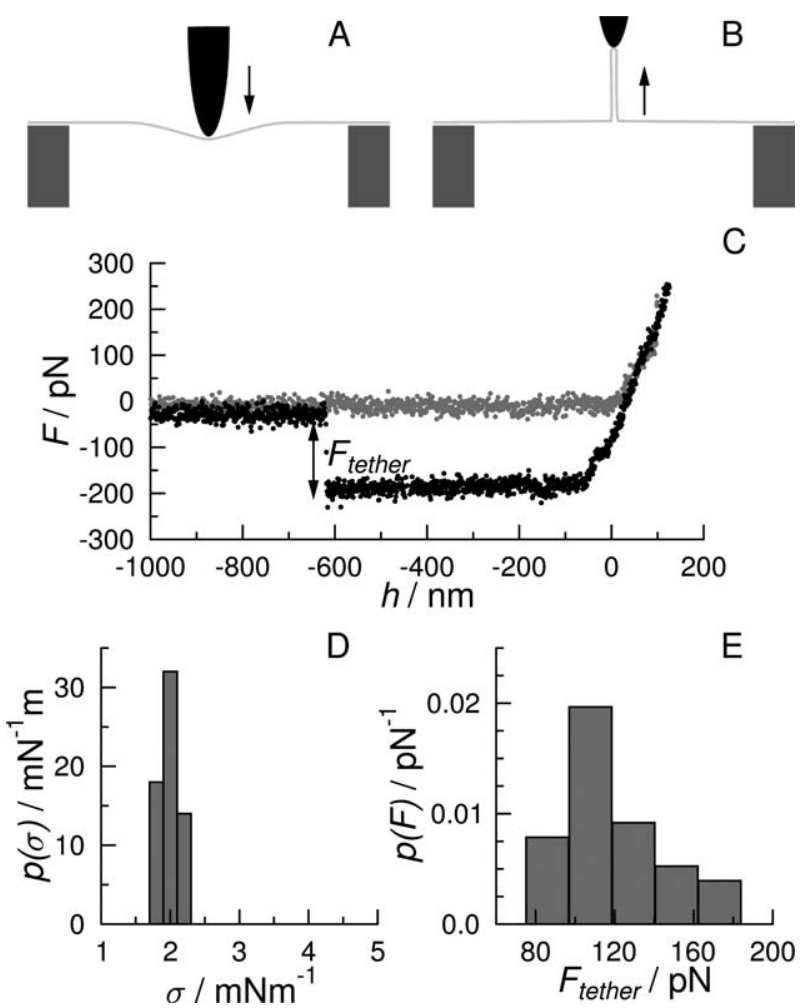

Fig. 5 Determination of tension and bending from one force cycle on supported pore-spanning membranes. ${ }^{30}$ (A and B) Typical force cycle of indentation (A) and retraction (B). Due to the interaction between the membrane and AFM tip a lipid tether is pulled from the membrane. (C) Typical force separation curve showing a linear force response upon indentation of the pore-spanning membrane (light gray) and formation of a nanotube upon retraction (dark gray) giving rise to a constant force plateau $\left(F_{\text {tether }}\right)$. Histograms of lateral tension $\sigma(D)$ and tether force $F_{\text {tether }}(\mathrm{E})$ obtained from force cycles on pure POPC.

assemble into polymeric vesicles in aqueous environments. ${ }^{44}$ Such polymerosomes are discussed as drug delivery agents, as they show many functions of biological membranes, e.g. membrane fusion and protein insertion while it is possible to set up specific properties like bending rigidity, thermal stress, tension resistance, and biological compatibility. ${ }^{44}$ Giant unilamellar polymerosomes made of $\mathrm{PB}_{130}-b-\mathrm{PEO}_{160}$ were studied by pipette suction experiments ${ }^{45}$ as well as supported porespanning membranes ${ }^{10}$ on porous arrays with pore diameters of $1.2 \mu \mathrm{m}$ prepared by spreading of giant polymerosomes. With both techniques the mechanical properties were analyzed. Pipette suction experiments require large vesicles and information is obtained from large areas; in contrast results from pore-spanning membranes are obtained on smaller length scales, defined by the pore size of the underlying array.

Fig. 6A shows a fluorescence image of a diblock-copolymer membrane hydrophobically loaded with Nile Red dye prepared on unfunctionalized porous arrays. In an AFM image scanned with a loading force of $2 \mathrm{nN}$ (Fig. 6B) the tip is able to penetrate uncovered pores approx. $400 \mathrm{~nm}$ deep while for covered pores the maximum indentation depth is approx. $250 \mathrm{~nm}$ which is shown by the cross-section in Fig. 6C.

The AFM tip is positioned at the center of the centrosymmetric membrane and then extended to indent the membrane into the 
pore to measure its response. During indentation and retraction of the AFM tip the restoring force acting on the cantilever is measured and plotted against the tip position. An example of such a force indentation experiment is depicted in Fig. 6D. Indenting the membrane into the pore leads to a non-linear response over several hundred nanometres without any rupture events (Fig. 6D) confirming the high yield force of polymer membranes to the imposed stress by a rather soft response. The indentation of the pore-spanning membrane is reversible and can be done several times without damaging the membrane.

As described above the response of a pore-spanning polymer membrane on pores with $1.2 \mu \mathrm{m}$ diameter may be only governed by pre-tension and stretching of the membrane. Tension would, in principle, lead to a linear response, while the cubic dependency in eqn (3) resembles the experimental data more. A linear combination of eqn (3) and an approximation of eqn (2) consider prestress $\sigma$ and stretching as the sources for the restoring forces and describe the force indentation experiment best. By fitting this linear combination to the measured data an excellent agreement between measured data and fitting results is obtained (white line in Fig. 6D). The mean pre-stress $\sigma$ was found to be $8.56 \mathrm{~N} \mathrm{~m}^{-1}$ and the elastic modulus $E$ ranged from 1.68 to $8.65 \mathrm{MPa}$ depending on the indentation velocity. A hysteresis between indentation and retraction is obvious from Fig. 7A indicating energy loss during the force cycle accompanied by a dependence on the indentation velocity. To account for viscoelastic contributions the pure elastic terms in eqn (2) and (3) are extended by a Kelvin-Voigt model. ${ }^{10}$ Applying the viscoelastic model to the obtained data a relaxation
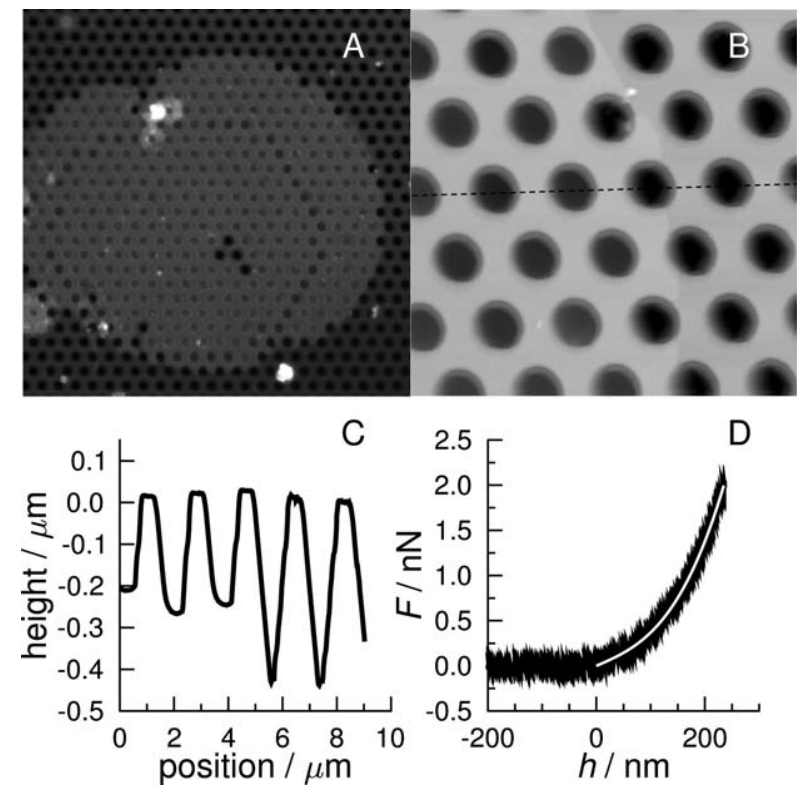

Fig. 6 Fluorescence and AFM images of supported pore-spanning polymer membranes and results of force indentation experiments obtained on porous arrays with a pore radius of $600 \mathrm{~nm} .{ }^{10}$ (A) Confocal laser scanning microscopy (CLSM) image of a polymer membrane spread on a porous silicon surface. (B) Contact mode AFM image of part of the membrane patch shown in (A). (C) The height profile of covered and uncovered pores along the dashed line marked in (B). (D) Force indentation curve (black) performed at $2 \mu \mathrm{m} \mathrm{s}^{-1}$ and $2 \mathrm{nN}$ indentation force. The white line is a fit using a linear combination of eqn (3) and an approximation of eqn (2).
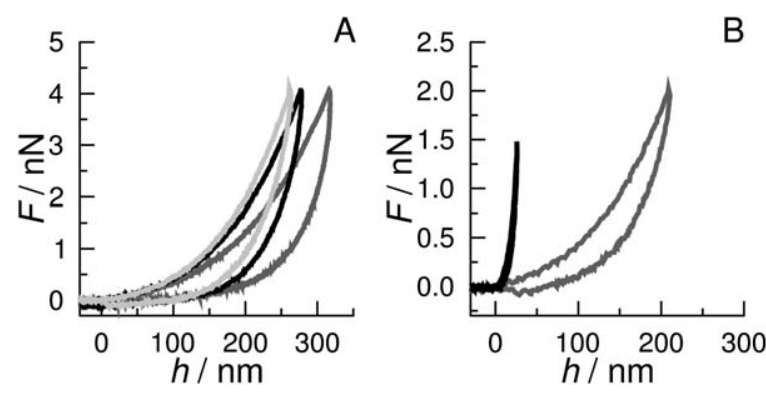

Fig. 7 Force cycles on pore-spanning $\mathrm{PB}_{130}-b$ - $\mathrm{PEO}_{160}$ membranes (A) performed with varying velocities (light gray: $0.5 \mu \mathrm{m} \mathrm{s}^{-1}$, black: $2 \mu \mathrm{m} \mathrm{s}^{-1}$, dark gray: $5 \mu \mathrm{m} \mathrm{s}^{-1}$ ). (B) Overlay of two force indentation curves performed on cross-linked (black) and native (gray) $\mathrm{PB}_{130}-b-\mathrm{PEO}_{160}$ membranes. The force cycles were performed at $2000 \mathrm{~nm} \mathrm{~s}^{-1} .^{10}$

time of $0.33 \mathrm{~s}$ was found, while the area compressibility ranges from 0.03 to $0.156 \mathrm{~N} \mathrm{~m}^{-1}$. These findings are in good accordance with pipette suction studies. ${ }^{46}$

The high stability and the possibility to localize a membrane patch with fluorescence and AFM imaging make it possible to measure the same pore-spanning membrane before and after any kind of treatments, like chemical modification. In the case of the $\mathrm{PB}_{130}-b$ - $\mathrm{PEO}_{160}$ diblock-copolymer membranes were crosslinked by UV-light. Comparing non-cross-linked and crosslinked membranes demonstrates that the cross-linked membranes are stiffer with less hysteresis (Fig. 7B).

Analysis of the cross-linked membranes resulted in an increase of the area compression modulus by more than a factor of ten to a range from 25 to $64 \mathrm{~N} \mathrm{~m}^{-1}$. Notably, the surface viscosity of non-cross-linked membranes is $0.005 \mathrm{~Pa} \mathrm{~m} \mathrm{~s}$ while in the crosslinked case, it increases by a factor of four to $0.02 \mathrm{~Pa} \mathrm{~m} \mathrm{~s}$. The results of modelling the response of polymer membranes upon indentation and the determined material properties are summarized in Table 2 .

\section{Phase separation of pore-spanning lipid membranes}

Apart from pure mechanical analysis of free-standing bilayers, pore-spanning membranes allow us to study phenomena like phase separation in lipid bilayers in an environment mimicking the plasma membrane of cells. Cellular membranes are an inhomogeneous mixture of various lipids and proteins, which are partly pinned to the underlying cytoskeleton. Phase separation in cellular membranes leads to the formation of nanodomains, ${ }^{47}$ while the investigation of phase separation phenomena in classical membrane systems like giant unilamellar vesicles or solid supported membranes shows domain formation on the micrometre scale. ${ }^{48}$ Orth et al. ${ }^{15}$ found that domain formation in pore-

Table 2 Summary of the properties of native and cross-linked polymer membranes. 'A' denotes results obtained from the viscoelastic model, while ' $\mathrm{B}$ ' refers to results obtained by fitting a linear combination of eqn (3) and an approximation of eqn (2) resembling a pure elastic model, to the data ${ }^{10}$

\begin{tabular}{lllll}
\hline $\begin{array}{l}\mathrm{PB}_{130}-b-\mathrm{PEO}_{160} \\
\text { membrane }\end{array}$ & $\eta_{\mathrm{eff}}^{\mathrm{A}} / \mathrm{Pa} \mathrm{m} \mathrm{s}$ & $\tau^{\mathrm{A}} / \mathrm{s}$ & $E^{\mathrm{B}} / \mathrm{MPa}$ & $K_{\mathrm{a}}^{\mathrm{A}} / \mathrm{N} \mathrm{m}^{-1}$ \\
\hline $\begin{array}{l}\text { Native } \\
\text { Cross-linked }\end{array}$ & 0.005 & 0.333 & 1.68 to $8.65 \times 10^{6}$ & $0.03-0.16$ \\
\hline
\end{tabular}


spanning membranes depends on the geometric properties of the porous array and therefore the domain size can be tuned by the size of the pores. Thus, pore-spanning membranes on a regular array of nano- to micrometre-sized pores seems to be a more suitable model system compared to classical solid supported membranes or vesicles to gain insight into the process and dynamics of phase separation in biological membranes. Vesicle spreading was used to form hybrid pore-spanning membranes for the investigation of phase separation in lipid bilayer mixtures composed of DOPC/sphingomyelin/cholesterol/ $\mathrm{G}_{\mathrm{b}} 3(40: 35: 20: 5)$ doped with the fluorescently labelled lipid Bodipy-PC. This lipid mixture phase separates into a liquidordered and a liquid-disordered phase as visualized by the fluorescence image in Fig. 8A. The fluorescently labelled lipid is excluded from the liquid ordered phase and these remain as dark spots in the membrane. The mobility of the whole lipid membrane was found to be maintained employing FRAP analysis. ${ }^{15,31} 75 \%$ of the domains are located near the pore rims, while $25 \%$ of the domains are located in the pore center. The overall fraction of the liquid-ordered domain varies between 25 and $35 \%$. This indicates that single lipids can diffuse freely in and out of a domain, while the diffusion of the whole domain is hindered keeping it in one pore-spanning membrane. The most interesting finding is the dependence of the domain size on the size of the underlying pores. As the pore size increases, the median domain size also increases, which is attributed to the underlying mesh, and not to the mechanical properties of the membrane. The membrane exhibits a constant lateral tension, which does not depend on the pore radius. While for pores of $2 \mu \mathrm{m}$ in diameter a domain area of $0.92 \pm 0.69 \mu \mathrm{m}^{2}$ was found, the areas decrease to $0.25 \pm 0.17 \mu \mathrm{m}^{2}$ and $0.1 \pm 0.05 \mu \mathrm{m}^{2}$ for pores with $1.2 \mu \mathrm{m}$ and $0.8 \mu \mathrm{m}$ diameters (Fig. 8B) respectively. The shape fluctuations of the domains show circularities between 0.8 and 1 as expected
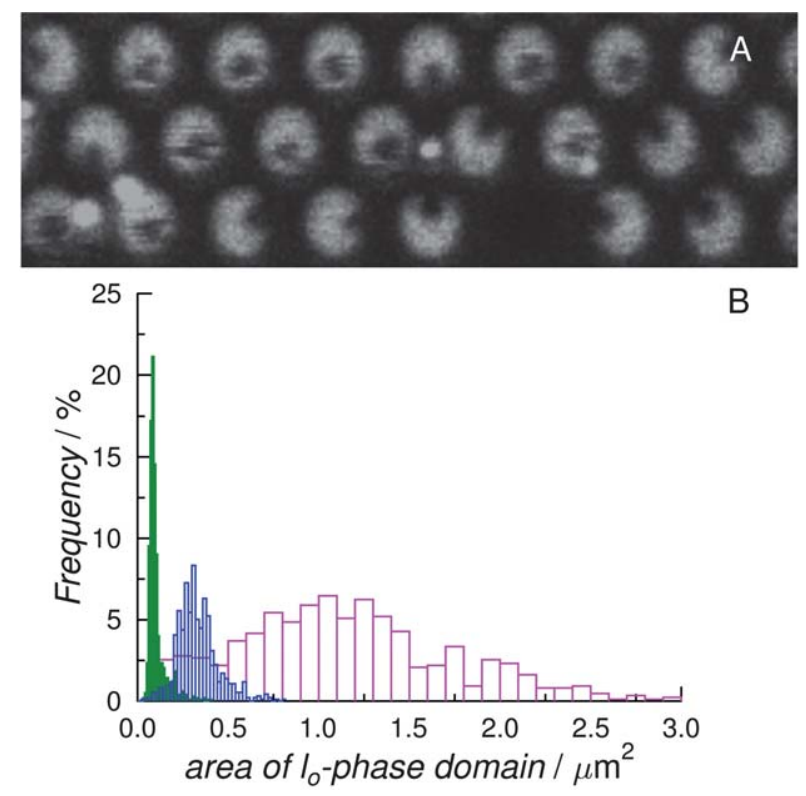

Fig. 8 Phase separation of hybrid pore-spanning lipid bilayers. (A) BodiPy-PC fluorescence of hybrid, phase separated pore-spanning membranes on pores with a diameter of $2 \mu \mathrm{m}$. (B) Area distribution of liquid ordered domains in pore suspending membranes (green: $R_{\text {pore }}=$ $0.4 \mu \mathrm{m}$, blue: $R_{\text {pore }}=0.6 \mu \mathrm{m}$, magenta: $\left.R_{\text {pore }}=1 \mu \mathrm{m}\right){ }^{15}$ from the minimization of line tension. Furthermore, phase separation could be reversed by removal of cholesterol with methyl- $\beta$-cyclodextrin resulting in a homogeneous fluorescence of the pore-spanning lipid membrane. In summary, the results demonstrate that pore-spanning membranes are a suitable model system in biophysics to partially mimic the influence of the cytoskeleton on domain formation in membranes. ${ }^{49}$

\section{Applications of porous arrays for sensors and drug release}

Besides the investigation of membrane model systems to mimic cellular membranes, pore arrays have gained widespread interest as biosensors. ${ }^{11,13}$ Successful separation of drug enantiomers ${ }^{50}$ and RNA/DNA oligomers ${ }^{51}$ are reported as well as studies on targeted drug release ${ }^{13}$ and protein detection. ${ }^{11,12}$ By employing a continuous lipid membrane covering the entire porous substrate, which is doped with a receptor, it is possible to study real time receptor ligand binding. ${ }^{14}$ Since the surface area of a porous substrate is generally one to three orders of magnitudes larger than the corresponding planar surface area, it is also useful for extracting proteins from cell lysates by employing receptordoped lipid membranes. ${ }^{14}$

Im et al. used surface plasmon resonance spectroscopy (SPR) on supported pore-spanning lipid bilayers on nanoporous arrays for the investigation of protein insertion and protein-antibody interaction. With SPR it is possible to observe the formation of supported pore-spanning membranes, the integration of a transmembrane protein e.g. $\alpha$-hemolysin $(\alpha-\mathrm{HL})$ and the binding of antibodies anti- $\alpha$-HL. This set-up combines the advantages of free-standing membranes for trans-membrane protein insertion by avoiding the insertion barrier at a solid support with SPR as a label-free measuring technique. This biosensor provides realtime kinetics for both, insertion of protein into the membrane and protein-antibody interaction. Mager and Melosh ${ }^{13}$ presented pore-spanning lipid bilayers to entrap material in nanopores, and its targeted release by the application of light. The picoliter-sized compartments, sealed by a lipid bilayer, are stable over weeks and the content release can be initialised by shedding light on small areas of a few tens of pores. Lazzara et al. ${ }^{11}$ employed porespanning lipid bilayers on AAO substrates to create attoliter-sized compartments, which were used not only for entrapment but also for exclusion of material. AAO substrates are optically transparent and confocal fluorescence microscopy can not only be used to visualize the membrane on top of the pores but fluorescent molecules inside the compartments can also be detected as displayed by $z$-stacks in Fig. 9A and B. The pore-spanning membrane emits red fluorescence, which is only visible on top of the porous substrate. In Fig. 9A, the membrane is used to exclude fluorescently labelled proteins from the pores and shows the adsorption of avidin to non-functionalized and uncovered areas of porous AAO substrates. The green avidin fluorescence is visible over the whole substrate when the pores are not sealed, while sealed areas remain black indicating the separation of the pore volume by a membrane. Trapping of a water soluble dye (pyranine) inside a compartment is also possible as depicted in Fig. 9B, where the membrane-covered pores are filled with a dye, while uncovered areas remain black. 


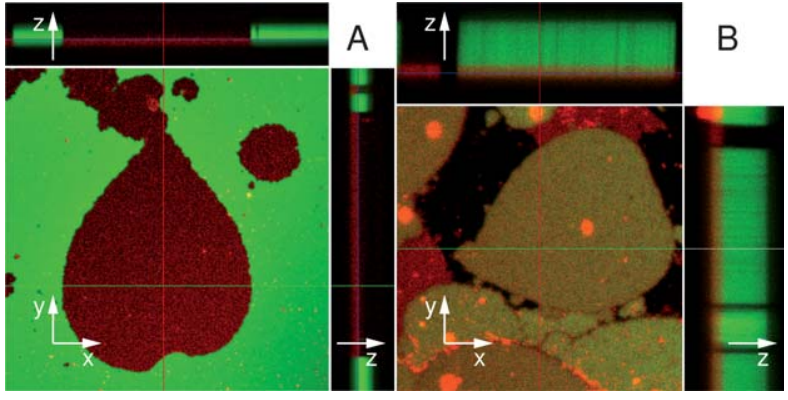

Fig. 9 Separation of attoliter sized compartments employing porespanning lipid bilayers. ${ }^{25}$ (A) Composite $z$-stack images (total $z$-distance: $15 \mu \mathrm{m}$ ) of a pore-spanning POPC bilayer preventing avidin entrance into the underlying pores (black areas). Main image: lipid membrane is located at the interface between the AAO and the bulk solution, showing both, red fluorescence from the membrane and green avidin fluorescence in the overlay. The total image size is $67 \mu \mathrm{m}$ times $67 \mu \mathrm{m}$. Top and right side images are line profiles in the $z$-direction and clearly show that the membrane prevents the fluorescently labeled proteins from entering the pores. "(B) Z-stack image of the membrane encapsulating pyranine dye molecules. The image size is $34 \mu \mathrm{m}$ times $34 \mu \mathrm{m}$.

In a very fundamental study on porous substrates, Lazzara et al. ${ }^{11}$ used avidin as a probe to investigate the response time of AAO, which is a prerequisite for biosensor applications. A possible flow cell geometry is depicted in Fig. 10A. A laminar flow with maximum velocity $u_{\max }$ in a channel of height $2 b$ runs along the porous substrate with length $L$. A probe such as a sticky protein is inserted into the flow and by optical waveguide spectroscopy (OWS) or fluorescence microscopy, the adhesion of the probe to the pore walls can be detected. If the bulk concentration of avidin is $10^{-3} \mathrm{M}$, roughly 3 molecules inside a pore with $R_{\text {pore }}=25 \mathrm{~nm}$ and $h=3 \mu \mathrm{m}$ equal the bulk concentration. Assuming a reasonable area of $5 \mathrm{~nm}^{2}$ for an avidin molecule nearly 20000 molecules can be adsorbed on the pore walls. The large surface area of the porous substrate provides nearly a perfect sink for the molecules, as every molecule which enters a pore is adsorbed immediately due to the large number of surface contacts of a diffusing molecule in a pore. A stationary Lévêque layer is produced by the depletion of the solution as shown by finite element (FE) calculations (Fig. 10B). ${ }^{23}$ Fig. 10C shows the normalized surface coverage $(\Theta(t))$ measured by OWS for AAO with different depths. The shorter the cavity the faster the saturation of the substrate is achieved. Compared to flat substrates it takes roughly hundred times longer and the kinetics show a linear behaviour as a function of time. According to simulations of the kinetics with varying $k_{\mathrm{ad}}$ and $k_{\mathrm{des}}=0$ the predominantly linear behaviour of the experimental kinetics could be reproduced (Fig. 10D) and flux into the pores was identified to be the governing effect, except for very low adsorption rate constants.

Porous substrates are suitable as sensors for molecular recognition, and for the investigation of transport processes. ${ }^{52-54}$ However, since flux to the pores is the limiting step, rate constants are not accessible from data obtained by adsorption into the pores. Release of material adsorbed on porous AAO substrates, however, allows for the determination of rate constants. Exchange of the bulk solution with an avidin-free solution, after the substrate had been saturated, leads to the
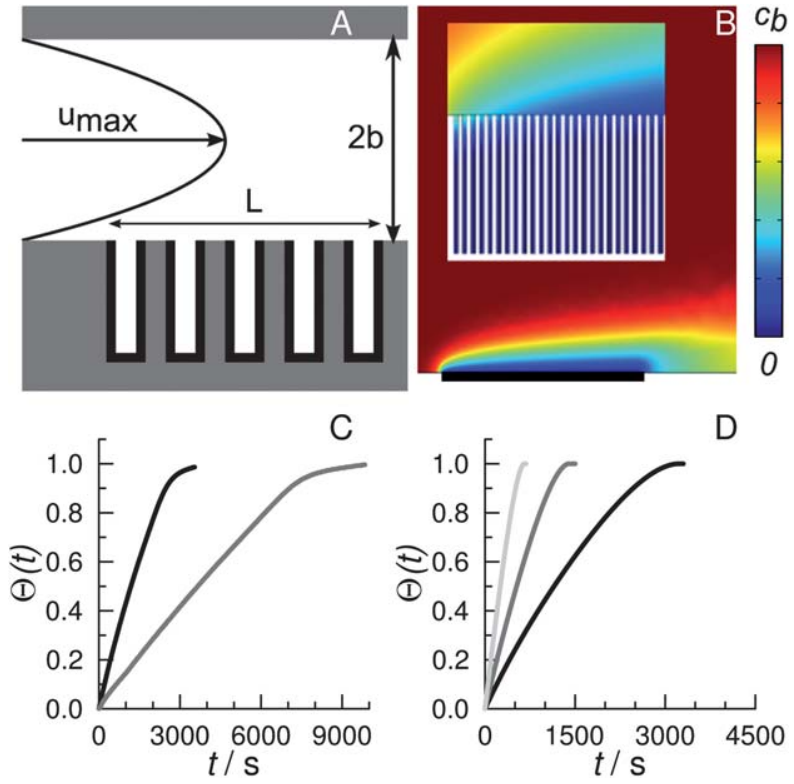

Fig. 10 Kinetic studies for the adsorption in porous materials. ${ }^{11}$ (A) Flow cell geometry used for finite element simulation and optical waveguide studies of protein adsorption. For the simulations, equally spaced pores with $R_{\text {pore }}=25 \mathrm{~nm}$ and $h=3.2 \mu \mathrm{m}$ along a distance of $L=120 \mu \mathrm{m}$ were used. The flow chamber has a height of $b=0.5 \mathrm{~mm}$ with a maximum flow velocity of $u_{\max }$. (B) Stationary concentration profile. (C) Avidin adsorption kinetics on AAO $\left(c_{\mathrm{b}}=1.5 \mu \mathrm{M}\right)$ as a function of pore depth $h$ ( $h=3.2 \mu \mathrm{m}$ (black), $h=6.4 \mu \mathrm{m}$ (gray)). (D) Surface coverage as a function of time for different pore lengths, $h=0.8 \mu \mathrm{m}$ (light gray), $h=1.6 \mu \mathrm{m}$ (gray), $h=3.2 \mu \mathrm{m}$ (black).

release of avidin from the pores as shown in Fig. 11A (gray curve). For short times, desorption of the material governs the kinetics, while for longer times the rate of adsorption contributes to the kinetics. An increase in $k_{\text {ad }}$ leads to longer desorption times due to rebinding as depicted by a finite element simulation in Fig. 11B. These kinds of release experiments are a way to elegantly determine the adsorption and desorption rate constants. By reducing the $\mathrm{pH}$ below the isoelectric point of the AAO, electrostatically bound avidin can be released, exhibiting

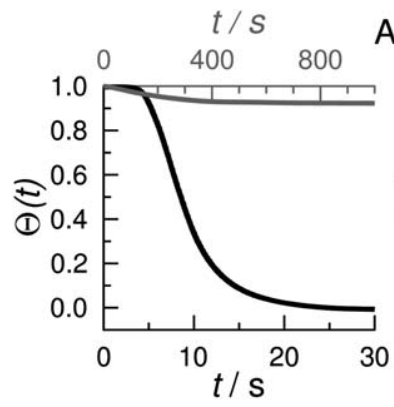

A

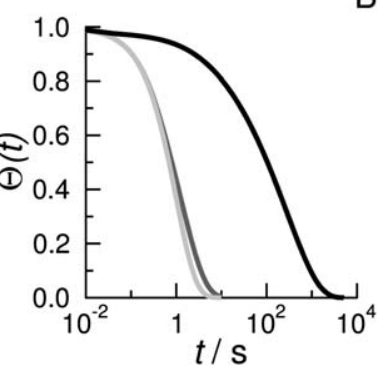

Fig. 11 Release of adsorbed material from porous arrays. ${ }^{11}$ (A) Experimentally measured surface coverage as a function of time for (i) the complete desorption of avidin from the porous surface by reduction of $\mathrm{pH}$ to values smaller than 3 (black curve) and (ii) the incomplete desorption when protein-free buffer is flushed onto the AAO after saturation of the avidin adsorption process (gray curve). (B) Simulated desorption kinetics as a function of $k_{\mathrm{ad}}\left(k_{\mathrm{ad}}=10^{3} \mathrm{~m}^{3} \mathrm{~mol}^{-1} \mathrm{~s}^{-1}\right.$ (light gray), $k_{\text {ad }}=1 \mathrm{~m}^{3} \mathrm{~mol}^{-1} \mathrm{~s}^{-1}$ (gray), $k_{\text {ad }}=10^{-3} \mathrm{~m}^{3} \mathrm{~mol}^{-1} \mathrm{~s}^{-1}$ (black)) with $k_{\mathrm{des}}=1 \mathrm{~s}^{-1}$. 
a much faster kinetics than by a simple flushing of the cell. Albeit rate constants are not accessible, porous AAO arrays might serve as an extraction unit. By modifying the whole surface with a phospholipid membrane (as described in Fig. 1D, bottom) doped with receptor lipids, specific proteins can be extracted from a cell lysate. ${ }^{14}$ Employing flow cell geometry as described, proteins can specifically bind to a receptor immobilized on the pore walls. As an example, we have used ezrin, a member of the ERM protein family, which binds to DOGS-NTA-Ni-doped membranes via its His-tag. OWS allows us to monitor the binding in situ. After adsorption of the protein and saturation is reached, the protein can be readily released by flushing with an imidazol-containing buffer.

\section{Conclusions}

Porous arrays are evolving as substrates for material testing and sensor applications especially in the field of biophysics. Fabrication of porous substrates is feasible on the nano- to micrometre scale from different materials with a variety of different surface functionalization strategies. These methods are a prerequisite for the preparation of pore-spanning membranes with tailored properties, such as tuned membrane tension and defined composition, which is the first step towards artificial plasma membranes. Pore-spanning membranes are suited to determine the mechanical properties of defined lipid compositions with high spatial resolution, which allows the investigation of spatial inhomogeneities. All membranes are stable in aqueous solutions and can be accessed from both sides. However, depending on the material, not every condition can be used, as, e.g., nanoporous alumina dissolves in an aqueous solution at low $\mathrm{pH}$ values. The large variety of surfaces and surface modifications allows for the construction of biosensors based on molecular recognition events and bilayer membranes. Owing to the large surface area, biosensors based on nanoporous arrays are very sensitive. However, the sensor response is hampered by the complicated kinetics when the molecules to be detected need to diffuse into the pores.

\section{References}

1 C. Hennesthal and C. Steinem, J. Am. Chem. Soc., 2000, 122, 80858086.

2 S. Steltenkamp, M. M. Müller, M. Deserno, C. Hennesthal, C. Steinem and A. Janshoff, Biophys. J., 2006, 91, 217-226.

3 I. Höfer and C. Steinem, Soft Matter, 2011, 7, 1644-1647.

4 E. Ovalle-Garca and I. Ortega-Blake, Appl. Phys. Lett., 2007, 91, 093901-1-093901-3.

5 C. Hennesthal, J. Drexler and C. Steinem, ChemPhysChem, 2002, 3, 885-889.

6 B. Lorenz, I. Mey, S. Steltenkamp, T. Fine, C. Rommel, M. M. Müller, A. Maiwald, J. Wegener, C. Steinem and A. Janshoff, Small, 2009, 5, 832-838.

7 I. Mey, M. Stephan, E. K. Schmitt, M. M. Müller, M. Ben Amar, C. Steinem and A. Janshoff, J. Am. Chem. Soc., 2009, 131, 7031-7039.

8 M. Kocun, T. D. Lazzara, C. Steinem and A. Janshoff, Langmuir, 2011, 27, 7672-7680.

9 R. P. Gonçalves, G. Agnus, P. Sens, C. Houssin, B. Bartenlian and S. Scheuring, Nat. Methods, 2006, 3, 1007-1012.

10 M. Kocun, W. Müller, M. Maskos, I. Mey, B. Geil, C. Steinem and A. Janshoff, Soft Matter, 2010, 6, 2508-2516.

11 T. D. Lazzara, I. Mey, C. Steinem and A. Janshoff, Anal. Chem., $2011,83,5624-5630$.

12 H. Im, N. J. Wittenberg, A. Lesuffleur, N. C. Lindquist and S.-H. Oh, Chem. Sci., 2010, 1, 688-696.
13 M. D. Mager and N. A. Melosh, Adv. Mater., 2008, 20, 4423-4427.

14 T. D. Lazzara, D. Behn, T.-T. Kliesch, A. Janshoff and C. Steinem, J. Colloid Interface Sci., 2012, 366, 57-63.

15 A. Orth, L. Johannes, W. Böhmer and C. Steinem, ChemPhysChem, 2012, 13, 108-114.

16 E. K. Schmitt, C. Weichbrodt and C. Steinem, Soft Matter, 2009, 5, 3347-3353.

17 S. Kresak, T. Hianik and R. L. C. Naumann, Soft Matter, 2009, 5, 4021-4032.

18 T. Fine, I. Mey, C. Rommel, J. Wegener, C. Steinem and A. Janshoff, Soft Matter, 2009, 5, 832-838.

19 M. Poot and H. S. J. van der Zant, Appl. Phys. Lett., 2008, 92, 063111.

20 C. Lee, X. Wei, J. W. Kysar and J. Hone, Science, 2008, 321, 385-388.

21 H. Masuda and K. Fukuda, Science, 1995, 268, 1466-1468.

22 G. E. Thompson and G. Wood, Treatise Mater. Sci. Technol., 1983, 23, 205-329.

23 T. D. Lazzara, T.-T. Kliesch, A. Janshoff and C. Steinem, ACS Appl. Mater. Interfaces, 2011, 3, 1068-1076.

24 F. Heinemann and P. Schwille, ChemPhysChem, 2011, 12, 2568-2571.

25 T. D. Lazzara, C. Carnarius, M. Kocun, A. Janshoff and C. Steinem, ACS Nano, 2011, 5, 6935-6944.

26 M. Busby, H. Kerschbaumer, G. Calzaferri and L. De Cola, $A d v$. Mater., 2008, 20, 1614-1618.

27 A. M. M. Jani, E. J. Anglin, S. J. P. McInnes, D. Losic, J. G. Shapter and N. H. Voelcker, Chem. Commun., 2009, 3062-3064.

28 M. J. Sailor and J. R. Link, Chem. Commun., 2005, 1375-1383.

29 J. Gong and X. Lin, J. Electrochem. Soc., 2004, 151, B519-B522.

30 M. Kocun and A. Janshoff, Small, 2012, 8, 847-851.

31 D. Weiskopf, E. K. Schmitt, M. H. Klühr, S. K. Dertinger and C. Steinem, Langmuir, 2007, 23, 9134-9139.

32 M. Böcker, S. Muschter, E. K. Schmitt, C. Steinem and T. E. Schäffer, Langmuir, 2009, 25, 3022-3028.

33 H.-J. Butt, B. Cappella and M. Kappl, Surf. Sci. Rep., 2005, 59, 1152.

34 D. Norouzi, M. M. Müller and M. Deserno, Phys. Rev. E: Stat., Nonlinear, Soft Matter Phys., 2006, 74, 061914.

35 E. Ovalle-Garcia, J. J. Torres-Heredia, A. Antillon and I. OrtegaBlake, J. Phys. Chem. B, 2011, 115, 4826-4833.

36 C.-H. Huang, P.-Y. Hsiao, F.-G. Tseng, S.-K. Fan, C.-C. Fu and R.-L. Pan, Langmuir, 2011, 27, 11930-11942.

37 N. Bhatia and W. Nachbar, Int. J. Nonlinear Mech., 1968, 3, 307-324.

38 M. R. Begley and T. J. Mackin, J. Mech. Phys. Solids, 2004, 52, 2005 2023.

39 H. Delano-Ayari, R. Al Kurdi, M. Vallade, D. Gulino-Debrac and D. Riveline, Proc. Natl. Acad. Sci. U. S. A., 2004, 101, 22292234.

40 J. Henriksen, A. Rowat, E. Brief, Y. Hsueh, J. Thewalt, M. Zuckermann and J. Ipsen, Biophys. J., 2006, 90, 1639-1649.

41 L. R. Arriaga, I. Lpez-Montero, F. Monroy, G. Orts-Gil, B. Farago and T. Hellweg, Biophys. J., 2009, 96, 3629-3637.

42 M. Maskos, Polymer, 2006, 47, 1172-1178.

43 A. Blanazs, S. P. Armes and A. J. Ryan, Macromol. Rapid Commun., 2009, 30, 267-277.

44 D. E. Discher and A. Eisenberg, Science, 2002, 297, 967-973.

45 H. Bermudez, A. K. Brannan, D. A. Hammer, F. S. Bates and D. E. Discher, Macromolecules, 2002, 35, 8203-8208.

46 B. M. Discher, Y.-Y. Won, D. S. Ege, J. C.-M. Lee, F. S. Bates, D. E. Discher and D. A. Hammer, Science, 1999, 284, 1143-1146.

47 C. Eggeling, C. Ringemann, R. Medda, G. Schwarzmann, K. Sandhoff, S. Polyakova, V. N. Belov, B. Hein, C. von Middendorff, A. Schonle and S. W. Hell, Nature, 2009, 457, 11591162.

48 S. L. Veatch and S. L. Keller, Phys. Rev. Lett., 2005, 94, 148101.

49 I. Weisswange, T. Bretschneider and K. I. Anderson, J. Cell Sci., 2005, 118, 4375-4380.

50 S. B. Lee, D. T. Mitchell, L. Trofin, T. K. Nevanen, H. Sderlund and C. R. Martin, Science, 2002, 296, 2198-2200.

51 I. Vlassiouk, A. Krasnoslobodtsev, S. Smirnov and M. Germann, Langmuir, 2004, 20, 9913-9915.

52 K.-P. S. Dancil, D. P. Greiner and M. J. Sailor, J. Am. Chem. Soc., 1999, 121, 7925-7930.

53 M. P. Schwartz, S. D. Alvarez and M. J. Sailor, Anal. Chem., 2007, 79, 327-334.

54 S. D. Alvarez, C.-P. Li, C. E. Chiang, I. K. Schuller and M. J. Sailor, ACS Nano, 2009, 3, 3301-3307. 